\title{
Natural-Setting PHR Usability Evaluation using the NASA TLX to Measure Cognitive Load of Patients
}

\author{
Emily Pachunka \\ University of Nebraska \\ Omaha \\ epachunka@unomaha.edu
}

\author{
John Windle \\ University of Nebraska \\ Medical Center \\ jrwindle@unmc.edu
}

\author{
Ryan Schuetzler \\ University of Nebraska \\ Omaha \\ rschuetzler@unomaha.edu
}

\author{
Ann Fruhling \\ University of Nebraska \\ Omaha \\ afruhling@unomaha.edu
}

\begin{abstract}
While personal health records (PHRs) carry an array of potential benefits such as increased patient engagement, poor usability remains a significant barrier to patients' adoption of PHRs. In this mixed methods study, we evaluate the usability of one PHR feature, an intake form called the pre-visit summary, from the perspective of cognitive load using real cardiovascular patients in a natural setting. A validated measure for cognitive load, the NASA Task Load Index, was used along with retrospective interviews to identify tasks within the pre-visit summary that increased participants' cognitive load. We found that the medications, immunizations, active health concerns, and family history pages induced a higher cognitive load because participants struggled to recall personal health information and also due to user interface design issues. This research is significant in that it uses validated measures of cognitive load to study real patients interacting with their PHR in a natural environment.
\end{abstract}

\section{Introduction}

Personal health records (PHRs) are private, secure, and confidential electronic applications that allow patients to access, manage, and share their health information [1]. PHRs tethered to their healthcare providers' electronic health records (EHRs), also known as patient portals, provide patients an online entryway to view their most up-todate personal health information including laboratory results, medication lists, immunization histories, and more [2]. Given their patient-centered nature, PHRs have the potential to increase patient engagement and enhance the medical decision-making process $[1,3-5]$.

Aside from simply viewing their health information, patients have the ability to also make updates and additions to their personal health information as well as perform several other functions within their PHRs. These features may include allowing patients to make payments, download educational materials, schedule or view upcoming clinic visits, and complete intake forms through their PHR [6]. One such intake form that is becoming a common part of patients' PHRs is the pre-visit summary. This patient intake form asks a patient to provide or update his or her health information prior to meeting with the clinician. It serves as an efficient means to engage the patients in reflecting upon their medical history and identifying new health concerns. The pre-visit summary promotes accurate update of information within the EHR and reduces the time required of clinic staff to update patients' records prior to being seen by their clinicians [3].

Despite the many prospective benefits and potential improved health outcomes associated with PHRs and the features they may offer, there are several barriers that impede PHR adoption. Traditional barriers of PHR adoption include privacy and security concerns, the "digital divide", and issues with data accuracy and integrity [1,7-10]. Yet, the main challenge for adoption and usage of PHRs and other health information technology is poor system usability $[8,11,12]$. Unfortunately, inefficient, complicated designs and ineffective user interfaces lead to dissatisfied users, whom often abandon such PHRs regardless of their potentially beneficial features [13].

The purpose of this study is to evaluate the usability of a newly designed online pre-visit summary from the perspective of cognitive load. Validated subjective measures of cognitive load were utilized with the aim of identifying specific tasks or design elements within the pre-visit summary that increase patients' cognitive load while completing the form in a natural setting. Participants (i.e. patients) were tasked to complete the pre-visit summary using their own personal health information, as this is the only realistic way to accurately measure cognitive load. To this end, this paper examines PHR usability with the intention 
to inform revisions of this pre-visit summary and to suggest methodology for evaluating patient-based technologies in a natural environment.

The remainder of this paper is as follows. The background section will formally define usability, present general methods for evaluating usability, and explore prior PHR usability studies. Next, the background will discuss cognitive load and techniques for its measurement. The purpose and guiding research question of this study are presented, which leads into the description of the research methodology implemented. The paper will conclude with results, discussion of the findings, limitations, and future research directions.

\section{Background}

\subsection{Usability of personal health records}

Previous work has identified the importance of usability to the adoption of PHR and EHR systems. The TURF (task, user, representation, function) framework was developed specifically to understand and evaluate the usability of EHR systems. According to the TURF framework, the term usability is defined as how "useful, usable, and satisfying a system is for the intended users to accomplish goals in the work domain" [14]. A system is said to be "useful" if it fully incorporate domain functions essential for work. The system is "usable" if it is easy to learn, efficient to operate, and error-tolerant. And lastly, the system is thought to be "satisfying" when it takes into account the users' impressions regarding likeability of the system. The dimensions and constructs of the TURF framework have served to guide the methodology and design of this study.

Usability testing can be accomplished via several approaches. Time per task, error rate, and completion time are a few examples of performance measures that are commonly examined in usability testing $[15,16]$. Another approach to evaluating a system's usability is a cognitive workload assessment, in which participants' cognitive load flucutations are analyzed to determine components of a system that may unnecessarily increase cognitive load. A formal definition of cognitive load is given below along with measurement techniques. Finally, most, if not all, usability assessments utilize subjective measures of usability that ask the representative users for their perceptions and opinions of the system [17]. Subjective measures for system usability may involve written questionnaires or surveys, think-aloud protocol, and retrospective interviews. One validated tool for evaluating overall system usability is the
System Usability Scale (SUS) by Brooke [18]. In the think-aloud method, the users are requested to verbally share their thoughts and concerns while performing tasks using the system; however, the validity and reliability of this method have been criticized as it may disrupt the participants' cognitive processes, and thus, may impact their overall performance [11]. Retrospective interviews are one alternative to the think-aloud method as such interviews still offer participants the opportunity to share their experiences and thoughts without interrupting their tasks. Too, retrospective interviews encourage the participants to make comments on usability issues that may not be directly related to the performed tasks [19].

Several prior studies have identified common usability issues with respect to PHRs. Segall et al. (2011) found that many patients reported issues with navigation, had difficulty searching for and entering data due to misleading interfaces, and struggled with unclear medical terminology. The participants, who were mostly cardiovascular patients with chronic illnesses, were pleased with the existing features within in the PHR; however, they felt that the PHR was not a "walk-up-and-use" system due to many usability issues [13]. Haggstrom et al. (2011) reported similar findings in evaluating a PHR for the VA in that the participants of their study also struggled with navigation and phrasing of medical jargon [20]. Thus, formal usability evaluations of HIT solutions are widely recognized as a crucial step to the success of these interactive information systems [11]. However, there seems to be a gap in the literature with respect to natural-setting PHR usability evaluations in which actual patients are using their own health information to organically complete tasks within a PHR. In our study, we try to address this gap by conducting usability assessment in such a way that real patients are completing an online pre-visit summary using their own health information while at the clinic for an actual provider visit.

\subsection{Cognitive load theory}

Cognitive Load Theory was developed in the 1980 s to provide guidance in optimizing information presentation and design to promote intellectual performance [21]. Formally, the theory defines cognitive load as "the load that performing a task imposes on the cognitive system of a learner" [22]. The theory makes assumptions regarding cognitive architecture including the premise that human is only able to handle two or three items of information simultaneously in working memory [22,23]. An increase in the number of items being stored or 
processed in working memory is then said to increase the cognitive load. If the task demand in working memory exceeds the available cognitive capacity, this results in cognitive overload, which ultimately has negative effects on learning and overall performance $[22,24,25]$. Cognitive load can be optimized through effective presentation of material in an attempt to develop schema, which are cognitive structures that are developed through experience and guide future cognitive processing [24].

While there is currently no consensus or "gold standard" for measuring cognitive load, it has been frequently shown that utilization of both objective and subjective measures provides a more accurate measurement of cognitive load [15,24,26-29]. Objective measures of cognitive load will either be performance-based or physiological measurements. One's performance can be evaluated using metrics such as error rate, time on task, overall speed, and task success [14,30,31]. Physiological measures assume that changes in cognitive processing are reflected in normal bodily functions [22,32,33]. Examples of physiological measures include heart rate, blood pressure, respiration rate, muscle tension, hormone levels, skin conductivity, pupil diameter, eye movements, and brain activity $[15,34]$.

Subjective measures of cognitive load reflect the assumption that subjects are able to report their cognitive processes by means of introspection [22,32]. Several valid, reliable tools currently exist to measure total cognitive load or at least a single construct of cognitive load [24,34-37]. These tools include the mental effort scale developed by Paas [35], the mental demand scale developed by Kalyuga et al [38], and the NASA Task Load Index (NASA TLX) [39]. Such subjective measures have been shown to be effective methods for measuring cognitive load $[34,37,40]$ and may have the ability to differentiate between cognitive load factors [27].

\subsection{Purpose of this study}

The purpose of this exploratory pilot study is to evaluate the usability of an online pre-visit summary from the perspective of patients' experienced cognitive load. A convergent mixed methods design was used to evaluate patients' cognitive load using validated measures in a natural setting, in which real heart and vascular patients were asked to complete an online pre-visit summary using their own health information. We asked the following research question to guide our methodology and analysis: Which tasks within the pre-visit summary induce an increased cognitive load upon patients completing the form?

\section{Research Method}

\subsection{Participant population}

Fourteen adult cardiovascular patients were recruited to be participants in this study. We chose to target patients with cardiovascular comorbidities because of the considerable disease burden of cardiovascular conditions on US healthcare. The American Heart Association's Heart Disease and Stroke Statistics Update in 2017 revealed that cardiovascular disease accounts for almost 1 in 3 deaths in the US, with heart disease being the number one cause of death in the nation. Further, it has been suggested that PHRs may hold the greatest value for patients with chronic diseases, as these patients require continuous care and long-term follow up [4].

\subsection{The pre-visit summary}

In this study, the online pre-visit summary being evaluated is part of a larger PHR prototype that was developed by the Cardiovascular Medicine Research group at a major medical institution. A multidisciplinary team including several subjectmatter experts (e.g. cardiologists, human-computer interaction experts, health informaticians, nurses) determined the appropriate content within this previsit summary as well as directed the progress of the user interface and functionality. This pre-visit summary consists of the following 10 pages designed to collect specific health information from cardiovascular patients: concerns to discuss, medications, medication allergies, other allergies, immunizations, past medical history, surgical/ procedure history, active health concerns, social history, and family history. In this study, each page of the pre-visit summary is considered one task. Participants were asked to complete all 10 tasks using their own health information in an attempt to accurately measure their cognitive load in a natural environment.

\subsection{Modified NASA TLX}

We chose to use the NASA Task Load Index (NASA TLX) to subjectively, quantitatively measure participants' cognitive load in this study given its multi-dimensional nature and its use within the healthcare and health information technology domains [41-46]. This validated instrument yields a computed cognitive load score based on either raw or weighted averages of a subject's rating across the following six 
subscales: mental demand, physical demand, temporal demand, performance, effort, and frustration [39]. For the purpose of this study, a modified version of the NASA TLX was administered to participants (Table 1). The mental demand, temporal demand, effort, and frustration subscales of the NASA TLX remain unchanged from its original version. However, the physical demand subscale was removed as this study is not intended to fluctuate or measure the participants' physical demands. The only other revision that was made to the NASA TLX is the reversal of the extremes of the performance subscale, which is done to maintain consistency of the instrument from the participants' perspective; this change was accounted for in data analysis. There is support in the literature for these modifications within the HIT domain [41-43].

Table 1. Modifications to NASA TLX questionnaire

\begin{tabular}{|l|l|l|}
\hline \multicolumn{1}{|c|}{ Subscale } & \multicolumn{1}{c|}{$\begin{array}{c}\text { Rating } \\
\text { Extremities }\end{array}$} & \multicolumn{1}{c|}{ Modification } \\
\hline $\begin{array}{l}\text { Mental } \\
\text { Demand }\end{array}$ & Low to High & No modification \\
\hline $\begin{array}{l}\text { Physical } \\
\text { Demand }\end{array}$ & Low to High & Subscale removed \\
\hline $\begin{array}{l}\text { Temporal } \\
\text { Demand }\end{array}$ & Low to High & No modification \\
\hline Performance & $\begin{array}{l}\text { Good to } \\
\text { Poor }\end{array}$ & $\begin{array}{l}\text { Rating extremities } \\
\text { reversed (i.e. Poor } \\
\text { to Good) }\end{array}$ \\
\hline Effort & Low to High & No modification \\
\hline Frustration & Low to High & No modification \\
\hline
\end{tabular}

\subsection{Procedures}

Each participant was tested individually in a private examination room after his or her clinic visit in the Heart and Vascular Center at a major medical center (Figure 1). Each testing session lasted approximately 30 minutes. The participant was informed about the goals and procedures of the study, and consent was obtained. As the participant completed each page within pre-visit summary using his or her own personal health information, the modified NASA TLX was administered to subjectively measure cognitive load for that task. A total of 10 NASA TLX questionnaires were obtained per participant, corresponding to the 10 tasks within the pre-visit summary. To conclude the testing session, a set of 5 open-ended interview questions were asked to allow the participant the opportunity to provide qualitative feedback regarding his or her experience in completing the pre-visit summary form. All study protocols were reviewed and approved by the university's IRB.

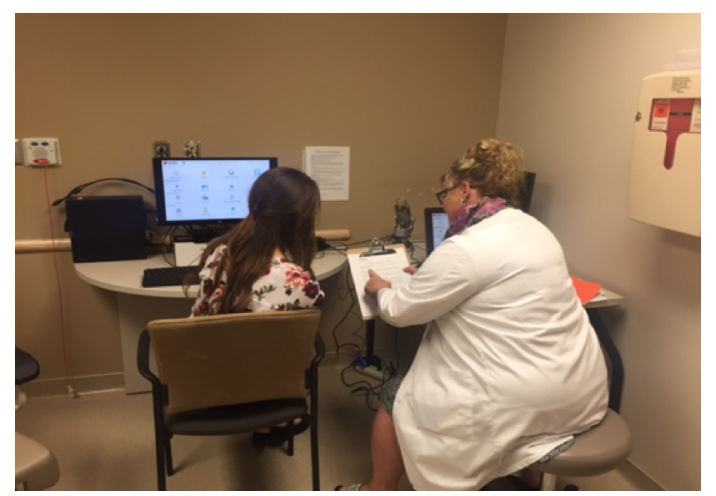

Figure 1. Testing Procedure and Set up

\section{Results}

\subsection{Participant characteristics}

A total of 14 adult patients of the Heart and Vascular Center were recruited and consented to participate in this study. One participant withdrew consent before completing the first page of the previsit summary due to difficulties using a mouse and keyboard. The remaining 13 participants completed the testing session in its entirety. Demographic data was obtained electronically prior to beginning the previsit summary, however, due to a technical malfunction, the demographic data for one participant was not saved. Thus, only NASA TLX data are available from this participant's testing session.

Table 2. Patient characteristics

\begin{tabular}{|c|c|}
\hline \multicolumn{2}{|l|}{ Sex } \\
\hline Female & 6 \\
\hline Male & 6 \\
\hline Age Range & 1 \\
\hline $19-40$ & 4 \\
\hline $41-64$ & 7 \\
\hline $65+$ & 0 \\
\hline "I am comfortable using a computer." \\
\hline Strongly disagree & 1 \\
\hline Disagree & 1 \\
\hline Neutral & 6 \\
\hline Agree & 4 \\
\hline Strongly agree &
\end{tabular}


Of the 12 participants with successfully saved demographic data, six were female (Table 2). One participant was between the ages of 19-40, four between ages 41-64, and the remaining seven patients were 65 years of age or older. The participants were also asked to rate their level of comfort using a computer on a 5-point Likert scale (Table 2), with 10 participants stating that they agreed or strongly agreed that they are comfortable using a computer.

\subsection{NASA TLX raw scores}

For each of the 13 participants that had completed the testing session, a single raw NASA TLX score was calculated for each of the 10 tasks in the pre-visit summary. A raw score ranges from $0-500$, with 500 meaning highest cognitive load. See [39] for description of the protocol to calculate raw NASA TLX scores. To visualize the data, a box plot was created to demonstrate trends of raw NASA TLX scores of the 13 participants for the 10 tasks (Figure 2). It is important to note that the goal of this study is not to achieve statistical significance but rather to explore trends and understand the interaction. Based on the median NASA TLX scores shown in the box plot, the tasks that induced the highest cognitive load are the medications, active health concerns, and immunizations pages (Figure 2). While the family history page did not exhibit a notably high median cognitive load, this task does appear to have induced cognitive load for some participants given that the upper quartile is the third highest behind medications and immunizations.

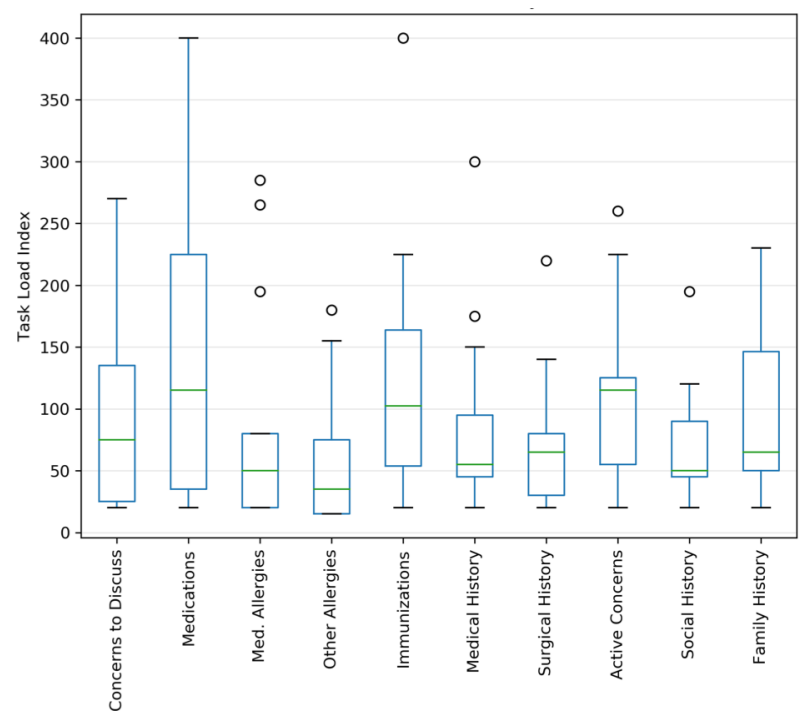

Figure 2. Raw NASA TLX scores
The tasks that induced the lowest cognitive load among participants are the medication allergies and other allergies pages. While there are a few outliers, the short height of these boxes seem to also indicate a higher level of agreement among the participants in terms of experienced cognitive load for these tasks.

\section{Discussion}

\subsection{High cognitive load tasks}

The purpose of this study was to evaluate the usability of the pre-visit summary by identifying tasks that induced a higher cognitive load upon participants. Using the NASA TLX, we have identified that the following four tasks seem to have increased participants' cognitive load: medications, immunizations, active health concerns, and family history. Interestingly, we have established internal consistency in our study as these quantitative results are in agreement with the qualitative data extracted from retrospective interviews with each participant after the completion of a testing session. When asked which tasks seemed to be the most difficult, nine of the thirteen participants stated that the medications page was most difficult. Four participants talked about their frustrations with the immunizations page, one participant responded with the active health concerns page, and three participants gave reasons why they struggled with the family history page. Based on the qualitative responses and our observations, we have identified two major themes to explain why these pages may have induced a higher cognitive load than the others: health information recall and user interface design issues.

\subsection{Health information recall}

The first theme deals with health information recall, which refers to mental retrieval of information from memory. Six of the participants that stated they felt the medications task was most challenging provided the reason that they have trouble remembering the names and dosages of their multiple medications. One patient said, "For me, it's more difficult to do the medications because I have so many of them."

Similarly, another participant shared that the medications page was difficult, "... because I take a lot of them and I am not sure what they do and how they affect me."

Five participants said that they would have completed this task more accurately and completely if 
they had a printed list of their medications to refer to while completing this page within the pre-visit summary. For example, one participant shared, "The one that frustrates me is medications. I have the list in a file... at home we would have that information."

Based on the NASA TLX scores, the immunization page also seemed to induce an increased cognitive load among participants. Four participants voiced their frustrations with the immunizations page during the retrospective interviews, three of whom explained that the challenges stemmed from having to remember details about the immunizations they have received. When the participant selected an immunization from the list, the pre-visit summary automatically would ask the user to provide the year in which he or she received that immunization. From our observations during the testing sessions, most participants struggled in recalling the dates of prior immunizations. One participant commented during the testing session, "I am just picking random years because I can't remember this."

With regard to information recall, the third task within the pre-visit summary that was mentioned during retrospective interviews was the family history page. Participants stated that it was a challenge remembering specific details regarding the health histories of their family members. One participant said, "... any of the health history of my family, I don't know any of that stuff.",

Another participant shared that the family history task was difficult, "... because it's not something I think about. I had to go back and think about what happened."

As mentioned previously, this pre-visit summary is intended to be part of patients' PHR for the Heart and Vascular Center. Ideally, the advantage of such PHR functionality is that patients would have the option to complete the pre-visit summary at home before arriving to the clinic for an appointment. Based on the responses from the retrospective interviews, most participants responded positively when we proposed that they would be able to complete the previsit summary and other intake forms at home. Most participants shared that they keep copies of their health records at home and would prefer to have access to such documentation when completing patient intake forms.

\subsection{Design issues}

The second major theme identified from the qualitative responses is related to user interface design issues within the pre-visit summary that may have unnecessarily induced a high cognitive load. While some of the cognitive load for the task was inherent in the task itself (e.g., recalling a list of medications), other load was introduced because of disconnect between patient expectations and system behavior. This type of cognitive load can be managed by aligning expectations and system behavior so that patients better understand how the system is behaving.

Three participants felt that the medications page was difficult due to design-related problems. For example, participants were asked to input and save their medications with the name, dosage, unit, and frequency of each medication. Once a medication was saved, the entry would be added to the ongoing list of medications saved on the page; however, multiple participants did not realize that their medications were in fact being saved and would try to add the same medication more than once (Figure 3). From our observations during the testing sessions, participants verbally expressed their frustration in completing the medications page because they did not see that their medications were being successfully saved. Like

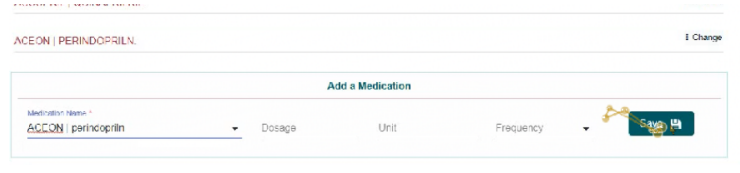

Figure 3. Medication entry

several other minor design issues throughout the form, one solution to this design issue on the medications page would be to improve feedback functionality and providing adequate error messages to avoid inaccurate or duplicate data.

Similarly, design issues appeared to the cause of confusion for three participants with the immunizations task. As mentioned above, several participants voiced difficulty in recalling the years in which they received specific immunizations. Participants seemed to consistently provide inaccurate years in order to complete the page. The system did not make it clear to participants that they could save an immunization in the system without a year. Thus, we would recommend that this portion of the pre-visit summary be revised to increase the visibility to users that years of immunizations are encouraged but not required. It is vital that we do not sacrifice accuracy of health information for ease of design, which is an important issue in the design of most if not all health information technology solutions.

The active health concerns page also arose in discussion during the retrospective interviews. In this section of the pre-visit summary, participants were tasked to check-box symptoms or health concerns by 
navigating through several body systems (e.g. cardiovascular, lungs, gastrointestinal, etc). One participant voiced that her confusion in completing this page stemmed from inconsistency in functionality. She said, "It was somewhat confusing because it looked like you could click on each thing, like lungs, and it would pull up different problems on there. But then, it would sorta just automatically drop down for you. I thought that was a little confusing."

With its current functionality, the form requires the user to click to the next body system once he or she has checked all applicable symptoms or concerns. However, if the user checks the box "No Active Concerns" (Figure 4), the form will automatically advance to the next body system. We believe this lack of consistency may have confused some participants and unnecessarily increased their cognitive load. Thus, we would suggest that revisions be made to the previsit summary to improve the consistency of the active health concerns page in this regard.

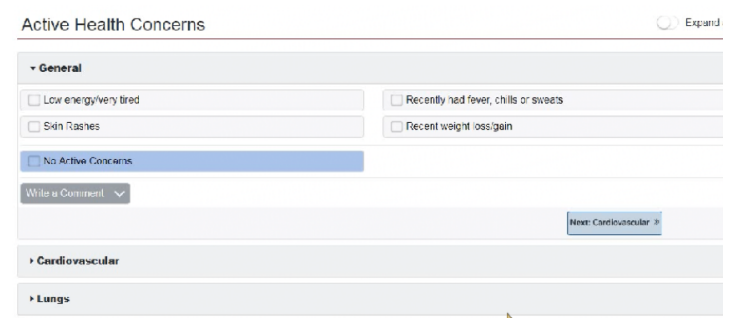

Figure 4. Active health concerns

\subsection{Low cognitive load tasks}

It was the goal of our research to identify those tasks that induced a higher cognitive load among participants completing the pre-visit summary. However, it is also important to identify the tasks that did not seem to induce a high cognitive load. The median raw NASA TLX scores indicated that the following tasks did not induce a high cognitive load: medication allergies, other allergies, past medical history, surgical history, and social history. None of these tasks were ever mentioned by participants during the retrospective interviews when asked about the tasks that were most difficult or confusing, which again exhibits internal consistency in our quantitative and qualitative results. We speculate that these pages were easier to complete for most participants as the information asked of the participants is more personal to patients. Further, allergic reactions, intolerances, diagnoses of medical conditions, and surgical procedures tend to be associated with specific events or time periods in patients' lives, which we hypothesize would make such information easier to recall.

Lastly, the median raw NASA TLX score for the concerns to discuss task seems to fall between that of high cognitive load and low cognitive load tasks. In this task, the participants were asked why they came to the doctor for the visit and what concerns they wanted to address. We suspect that the experienced high cognitive load on this initial task because this was the first exposure to the new PHR system. There also seemed to be mixed experiences in completing this tasks given the wide interquartile range shown in the box plot.

\subsection{Paper versus online}

Previous studies have found that electronic intake forms are more efficient and preferred by patients as compared to paper-based forms [47-50]. In the retrospective interview portion of the sessions, we also asked the participants if they would prefer to complete the pre-visit summary online as they did in the study or in a paper-based format. About $85 \%$ of participants $(11 / 13)$ responded that they would prefer the online format. Several reasoned that they preferred the online format because it would be more convenient to complete the pre-visit summary at home or prior to arriving to the clinic.

Of the remaining two participants, one responded that the paper-based pre-visit summary would be preferred and stated, "I would probably do better on the paper. "It is important to note that this participant was the only participant to respond with, "Disagree" when asked about the level of comfort with a computer (Table 2). The last participant preferred neither the paper nor online format as the preferred method given by this participant was verbal intake by medical staff.

\subsection{Limitations and future directions}

One limitation in our study is the small sample size. As this was a pilot study, evaluation of 13 participants' interactions we believe provided adequate assessment of the pre-visit summary. However, in future usability evaluations of the entire PHR prototype developed by the cardiovascular medicine research group at our medical university, we intend to target a larger sample size. We would also like to incorporate other measurements of cognitive load, like eye tracking, along with the NASA TLX to provide more insight into the induced cognitive load upon patients interacting with their PHRs.

As mentioned earlier, it has been found in prior research that PHRs may hold the greatest value for 
patients with multiple comorbid conditions. Patients following with multiple specialists for management of several comorbidities are very likely to have complex care plans that require many medications. As observed in our research, many participants experienced an increase in cognitive load when trying to complete the medications page, several of whom expressed their difficulty with information recall. According to a 2015 study conducted by Jones et al., only $22 \%$ of older adult patients were able to accurately recall the names of their medications, and only $35 \%$ could correctly associate the medications with their medical conditions [51]. Another future direction of our research deals specifically with optimizing the PHR to help draw associations between patients' medications with their medical comorbidities. Using foundations from cognitive load theory and findings from this study, we feel that we could optimize patients' experienced cognitive load by generating mental schema through the relation of a medication to a disease condition and the prescribing provider.

\section{Conclusion}

In this study, we identified several tasks within the pre-visit summary that induced an increased cognitive load upon cardiovascular patients including medications, immunizations, family history, and active health concerns. We highlighted two major themes to explain why these tasks within the pre-visit summary may have induced an increased cognitive load among participants. The first theme, health information recall, suggests that participants may have struggled remembering names of medications or details regarding immunizations because of difficulty understanding medical jargon. We found that participants did not experience high cognitive load completing pages in the pre-visit summary in which the information was perhaps more personal or related to particular events.

The second major theme that arose in the qualitative data dealt with user interface design. This research identified several issues with user interface design that we recommend be addressed in the next iteration of the pre-visit summary. Possible solutions to these problems include: 1) enhancing user feedback on the medications page to better inform the user once a medication was successfully added; 2) adjusting the granularity of error notifications to avoid duplicate medications; 3 ) increasing functional visibility on the immunizations page; 4 ) improving the consistency of the active health concerns page.

In the current study, we were able to establish internal consistency between raw NASA TLX scores and information extracted from retrospective interviews of the participants, suggesting that the NASA TLX was a successful subjective measure of cognitive load in evaluating patient-based health information technology. We intend to utilize both objective and subjective measures of cognitive load in our future work and incorporate more concepts of cognitive load theory to further understand the cognitive demands that health information technology may induce upon patients. We hope that through such efforts we can continue to optimize information technology used to support and engage patients in their medical care.

\section{References}

[1] Wang T, Dolezel D. Usability of Web-based Personal Health Records: An Analysis of Consumers' Perspectives Background and Significance. Perspect Heal Inf Manag 2016;13.

[2] Vydra Pressler T, Cuaresma E, Kretovics M, BoseBrill S. Diffusion and Use of Tethered Personal Health Records in Primary Care. Perspect Heal Inf Manag [Internet] 2015;(1):1-16. PMID:26755897

[3] Hummel J, Qualis Health Seattle M, Peggy Evans W, Bearden T, Health Q, Susan McBride I. Providing Clinical Summaries to Patients after Each Office Visit: A Technical Guide With contributions from. 2012; Available from: www.wirecQH.org

[4] Tenforde M, Jain A, Hickner J. The value of personal health records for chronic disease management: What do we know? Fam Med 2011; PMID:21557106

[5] Estabrooks PA, et al. Harmonized patient-reported data elements in the electronic health record: Supporting meaningful use by primary care action on health behaviors and key psychosocial factors. J Am Med Informatics Assoc 2012; PMID:22511015

[6] What is a patient portal? [Internet]. 2015 [cited 2017 Nov 10]. Available from:

https://www.healthit.gov/providersprofessionals/faqs/what-patient-portal

[7] Agarwal R, Angst CM. Technology-enabled transformation in U.S. health care: early findings of personal health records and individual use. HumanComputer Interact Manag Inf Syst Appl 6th ed Armonk: M. E. Sharpe; 2006.

[8] Alyami MA, Song Y-T. Removing barriers in using personal health record systems. 2016 IEEE/ACIS 15th Int Conf Comput Inf Sci 2016. [doi:

10.1109/ICIS.2016.7550810] 
[9] Buntin MB, Burke MF, Hoaglin MC, Blumenthal D. The benefits of health information technology: A review of the recent literature shows predominantly positive results. Health Aff 2011; PMID:21383365

[10] Clarke M, Sitorius M, Windle T, Fruhling A, Bernard $\mathrm{T}$, Windle J. A qualitative study of user-desired personal health record functionality: impact of age on desired PHR functionality. AMIA; 2016. AMIA 2016.

[11] Jaspers MWM. A comparison of usability methods for testing interactive health technologies: Methodological aspects and empirical evidence. Int J Med Inform 2009; PMID:19046928

[12] Showell C. Barriers to the use of personal health records by patients: a structured review. PeerJ 2017; PMID:28462058

[13] Segall N, Saville JG, Carlson B, Wright MC, Schulman K, Tcheng JE. Usability Evaluation of a Personal Health Record. AMIA Annu Symp 2011. p. 1233-1242.

[14] Zhang J, Walji MF. TURF: Toward a unified framework of EHR usability. J Biomed Inform 2011;44(6):1056-1067. PMID:21867774

[15] Lazar J, Feng JH, Hochheiser H. Research Methods in Human-Computer Interaction. Great Britain: John Wiley \& Sons Ltd; 2010. ISBN:978-0-470-72337-1

[16] Middleton B, et al. Enhancing patient safety and quality of care by improving the usability of electronic health record systems: Recommendations from AMIA. J Am Med Informatics Assoc 2013; PMID:23355463

[17] Rubin J. Handbook of Usability Testing: How to Plan, Design, and Conduct Effective Tests. New York: John Wiley \& Sons, Inc.; 1994. ISBN:0-471-59403-2

[18] Brooke J. SUS-A quick and dirty usability scale. In: Jordan PW, Thomas B, Weerdmeester BA, McClelland IL, editors. Usability Eval Ind London: Taylor \& Francis, Ltd.; 1996. p. 189-194.

[19] Bowers VA, Snyder HL. Concurrent versus retrospective verbal protocol for comparing window usability. Proc Hum Factors Soc 34th Annu Meet 1990;1270-1274.

[20] Haggstrom DA, Saleem JJ, Russ AL, Jones J, Russell SA, Chumbler NR. Lessons learned from usability testing of the VA's personal health record. J Am Med Informatics Assoc 2011; PMID:21984604

[21] Chandler P, Sweller J. Cognitive load theory and the format of instruction. Cogn Instr 1991;8(4):293-332.

[22] Paas FGWC, Van Merrienboer JJG. Instructional control of cognitive load in the training of complex cognitive tasks. Educ Psychol Rev 1994;6(4):351-371.
[23] Sweller J, van Merriënboer JJG, Paas FGWC. Cognitive architecture and instructional design. Educ Psychol Rev 1998;10(3):251-296.

[24] Paas FGWC, van Merriënboer JJG, Adam JJ. Measurement of Cognitive Load in Instructional Research. Percept Mot Skills 1994; PMID:7808878

[25] Sweller J. Cognitive Load During Problem Solving: Effects on Learning. Cogn Sci 1988;12:257-285. [doi: $\left.10.1207 / \mathrm{s} 15516709 \operatorname{cog} 1202 \_4\right]$

[26] Davids MR, Chikte UME. Optimising cognitive load and usability to improve the impact of e-learning in medical education. African J Heal Prof Educ 2015;7(2):147-152.

[27] Chen S. The construct of cognitive load in interpreting and its measurement. Perspect Stud Transl [Internet] Taylor \& Francis; 2017;25(4):640-657. [doi: 10.1080/0907676X.2016.1278026]

[28] Wang Q, Yang S, Cao Z, Ma Q. An eye-tracking study of website complexity from cognitive load perspective. Decis Support Syst 2014;62.

[29] Asan O, Yang Y. Using Eye Trackers for Usability Evaluation of Health Information Technology: A Systematic Literature Review. JMIR Hum Factors 2015; PMID:27026079

[30] Ayres P, Paas F. Cognitive load theory: New directions and challenges. Appl Cogn Psychol [Internet] 2012;26(6):827-832. PMID:83711451

[31] Schultheis H, Jameson A. Assessing cognitive load in adaptive hypermedial systesm: Physiological and behavioral methods. In: De Bra PME, Nejdl W, editors. Adapt Hypermedia Adapt Web-Based Syst Springer, Berlin, Heidelberg; 2004.

[32] Khawaja MA, Chen F, Marcus N. Measuring Cognitive Load Using Linguistic Features: Implications for Usability Evaluation and Adaptive Interaction Design. Int $\mathrm{J}$ Hum Comput Interact 2014; [doi: $10.1080 / 10447318.2013 .860579]$

[33] Kramer AF. Physiological metrics of mental workload: A review of recent progress. In: Damos D, editor. Mult Task Perform London: Taylor \& Francis; 1991.

[34] Antonenko P, Paas F, Grabner R, van Gog T. Using Electroencephalography to Measure Cognitive Load. Educ Psychol Rev 2010;22(4):425-438. PMID:814356653

[35] Paas FGWC. Training Strategies for Attaining Transfer of Problem-Solving Skill in Statistics: A 
Cognitive-Load Approach. J Educ Psychol

1992;84(4):429-434. [doi: 10.1037/0022-0663.84.4.429]

[36] Naismith LM, Cheung JJH, Ringsted C, Cavalcanti RB. Limitations of subjective cognitive load measures in simulation-based procedural training. Med Educ 2015;49(8):805-814. PMID:26152492

[37] Chen S, Epps J, Chen F. A comparison of four methods for cognitive load measurement. Proc 23rd Aust Comput Interact Conf - OzCHI '11 [Internet] 2011;76-79. [doi: 10.1145/2071536.2071547]

[38] Kalyuga S, Chandler P, Sweller J. Managing Splitattention and Redundancy in Multimedia Instruction. Appl Cogn Psychol 1999;13(4):351-371. PMID:11818227

[39] NASA. NASA Task Load Index (TLX) v. 1.0 Manual. 1986.

[40] Brünken R, Plass JL, Leutner D. Direct measurement of cognitive load in multimedia learning. Educ Psychol 2003;38(1):53-61.

[41] Longo L, Kane B. A Novel Methodology for Evaluating User Interfaces in Health Care. 24th Int Symp Comput Med Syst 2011

[42] Moacdieh N, Sarter N. Clutter in electronic medical records: examining its performance and attentional costs using eye tracking. Hum Factors 2015;57(4):591-606.

[43] Ariza F, Kalra D, Potts HWW. How do clinical information systems affect the cognitive demands of general practitioners? Usability study with a focus on cognitive workload. J Innov Heal Informatics J Innov Heal Inform J Innov Heal Informatics [Internet] 2015;2222(4):379-390. [doi: 10.14236/jhi.v22i4.85]

[44] Colligan L, Potts H, Finn C, Sinkin R. Cognitive workload changes for nurses transitioning from a legacy system with paper documentation to a commercial electronic health record. Int J Med Inform 2015;84(7):469476.

[45] Tubbs-Cooley HL, Mara CA, Carle AC, Gurses AP. The NASA Task Load Index as a measure of overall workload among neonatal, paediatric and adult intensive care nurses. Intensive Crit care Nurs 2018 Jun;46:64-69. PMID:29449130

[46] Yu D, Lowndes B, Morrow M, Kaufman K, Bingener J, Hallbeck S. Impact of novel shift handle laparoscopic tool on wrist ergonomics and task performance. Surg Endosc 2016;30(8):3480-90. PMID:26541720

[47] Howell M, Hood AJ, Jayne DG. Use of a patient completed iPad questionnaire to improve pre-operative assessment. J Clin Monit Comput 2017; PMID:26715416
[48] Lane SJ, Heddle NM, Arnold E, Walker I. A review of randomized controlled trials comparing the effectiveness of hand held computers with paper methods for data collection. BMC Med Inform Decis Mak. 2006. PMID: 16737535

[49] Martin P, et al. Patient preference: A comparison of electronic patient-completed questionnaires with paper among cancer patients. Eur J Cancer Care (Engl) 2016; PMID:25899560

[50] Salaffi F, Gasparini S, Ciapetti A, Gutierrez M, Grassi W. Usability of an innovative and interactive electronic system for collection of patient-reported data in axial spondyloarthritis: Comparison with the traditional paperadministered format. Rheumatol (United Kingdom) 2013; PMID:23955646

[51] Jones GT, Tabassum V, Zarow GJ, Ala TA. The Inability of Older Adults to Recall Their Drugs and Medical Conditions. Drugs and Aging 2015;32(4):329336. PMID:25829296 